\title{
Arthroscopic Bankart repair augmented by plication of the inferior glenohumeral ligament via horizontal mattress suturing for traumatic shoulder instability
}

\author{
Kong Hwee $\underline{L e}^{1}$, MBBS, MrCSEd, Henry Soeharno ${ }^{1}$, MBBS, MRCSEd, Chee Ping $\underline{C h e w}^{1}$, MBBS, MRCSEd, \\ Denny $\underline{\text { Lie }}^{1}$, FRCSEd, FAMS
}

INTRODUCTION This study aimed to evaluate the two-year clinical outcomes of arthroscopic Bankart repair augmented by plication of the inferior glenohumeral ligament (IGHL) via horizontal mattress suturing.

METHODS This study was a retrospective analysis of 76 arthroscopic Bankart repairs by a single surgeon from 2004 to 2008. Bioabsorbable suture anchors were used, with anchors placed at the 5:30, 4 and 3 o'clock positions of the right shoulder (correspondingly at the 6:30, 8 and 9 o'clock positions for the left shoulder). The lower most anchor was tied via horizontal mattress suture with plication of the IGHL, while the remaining two anchors were tied using simple sutures. The patients were postoperatively assessed, clinically and using the University of California Los Angeles (UCLA) shoulder rating scale.

RESULTS The mean age of the patients at the time of presentation was $24.09 \pm 7.98$ years, and the mean duration of follow-up was 28.7 months. The postoperative recurrence rate of dislocation was $7.89 \%$ (six shoulders), of which five shoulders required revision surgeries. Excluding the revision cases, clinical assessment of the strength of the supraspinatus and the range of motion of the operated shoulders at two years post operation were either maintained or full in all patients. Analysis of the UCLA results showed pre- and postoperative mean scores of $25.94 \pm 3.43$ and $33.77 \pm 3.07$, respectively $(p<0.05)$.

CONCLUSION Arthroscopic Bankart repair augmented by plication of the IGHL and anchored via horizontal mattress suturing is a safe and reliable treatment for shoulder instability, with good clinical outcomes and low recurrence rates.

Keywords: arthroscopic Bankart repair, horizontal mattress suture, inferior glenohumeral ligament, traumatic shoulder instability

\section{INTRODUCTION}

The shoulder joint is the most mobile joint in the body and the overall incidence of shoulder dislocation is about 17-24 in 10,000 persons per year, with $85 \%$ of these dislocations being anterior dislocations. ${ }^{(1,2)}$ Shoulder stability depends on both static and dynamic mechanisms. Anteroinferior shoulder instability is usually traumatic. A study by Brophy and Marx showed that compared to conservative management, surgical treatment results in superior outcomes that prevent recurrences in first-time anterior shoulder dislocation, particularly in young, active male patients. ${ }^{(3)}$ Open Bankart repair was reported to be the gold standard, with high success rates (> 90\%) and low recurrence rates $(<9 \%){ }^{(3)}$ However, open surgery is associated with significant morbidities such as longer hospitalisation period, greater blood loss, longer rehabilitation time and restricted range of motion, especially external rotation due to damage of the subscapularis. ${ }^{(4)}$

Advancements in surgical instrumentations and surgical techniques have led to the evolution of minimally invasive arthroscopic repair of Bankart lesions in the 1990s. ${ }^{(5)}$ The potential advantages of arthroscopic repair over open repair include a less extensive surgical dissection with a more comprehensive view of the operating field, less blood loss and pain, and faster rehabilitation with decreased subscapularis dysfunction. However, early arthroscopic techniques using staple capsulorrhaphy, transglenoid suturing and bioabsorbable tack fixations were reported to have higher recurrence rates than open Bankart repair. ${ }^{(6-8)}$ With the advent of suture anchors, the outcomes of arthroscopic repair have improved significantly and are able to match that of open repairs.

In the current literature, there is no report on the outcomes of arthroscopic Bankart repair augmented by plication of the inferior glenohumeral ligament (IGHL) secured with bioabsorbable suture anchors and horizontal mattress suturing. Thus, we aimed to introduce the aforementioned surgical technique, which shows potential in improving the outcomes of arthroscopic repair of Bankart lesions.

\section{METHODS}

After obtaining approval from the Institutional Review Board of Singapore General Hospital, the records of 75 patients who underwent arthroscopic Bankart repair for recurrent anterior glenohumeral instability by a single surgeon at the hospital were retrieved. One patient had bilateral shoulders repaired. The data was prospectively collected and a retrospective review of the data was performed. The subject inclusion criteria for this study were recurrent anterior glenohumeral subluxation or dislocation, and the presence of a Bankart

\footnotetext{
${ }^{1}$ Department of Orthopaedic Surgery, Singapore General Hospital, Singapore

Correspondence: Dr Denny Tjiauw Tjoen Lie, Senior Consultant, Department of Orthopaedic Surgery, Singapore General Hospital, Outram Road, Singapore 169608. denny.lie.t.t@sgh.com.sg
} 


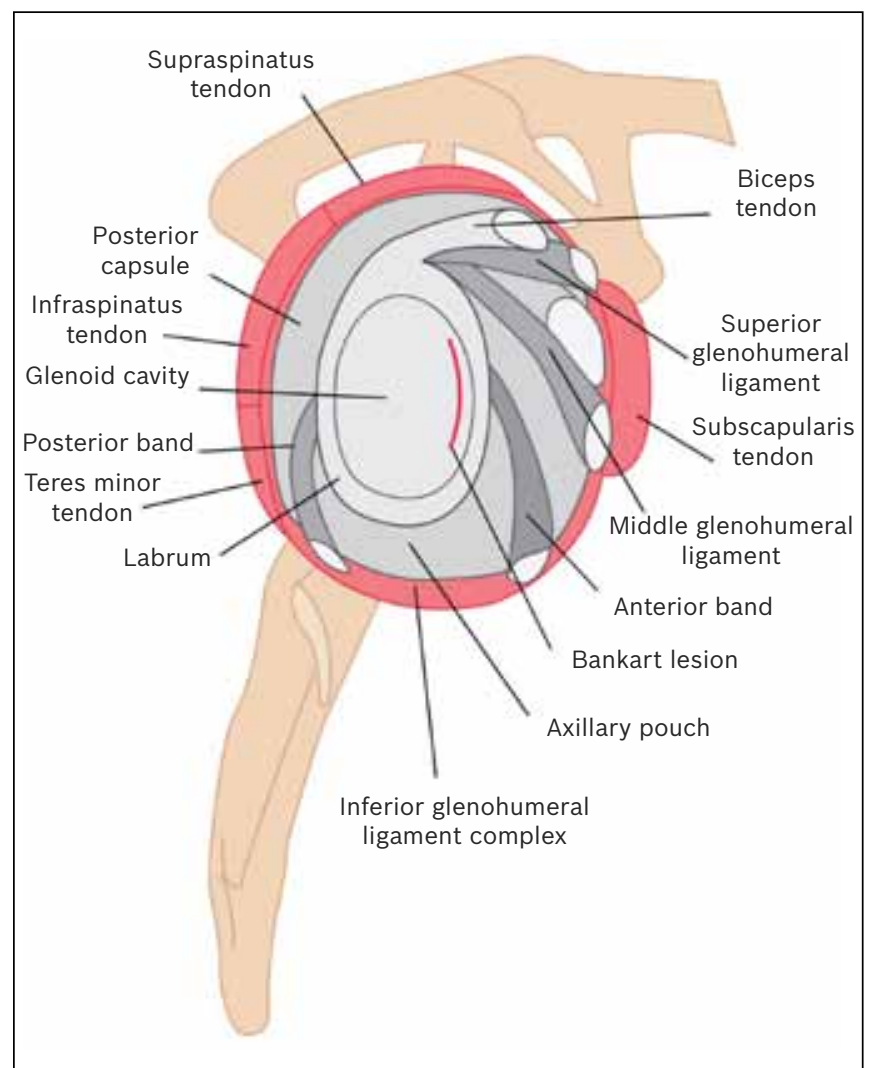

Fig. 1 Illustration shows a Bankart lesion and the structures surrounding it.

lesion confirmed on magnetic resonance (MR) arthrography or imaging. The exclusion criteria were posterior instability, multidirectional instability, presence of a Hill-Sachs lesion involving more than $25 \%$ of the humeral head, and presence of a bony Bankart lesion involving more than $25 \%$ of the glenoid. All patients demonstrated positive results in the apprehension, load and shift tests, and had preoperative radiographs (anteriorposterior, lateral, axillary and scapular-Y views) taken. The augmented arthroscopic Bankart repair technique introduced in this study is a novel surgical technique that aims to repair the detached anteroinferior labrum with bioabsorbable suture anchors, which are tied down with the anterior band of the IGHL via horizontal mattress and simple vertical suturings.

All the operations were performed by the same surgeon, who used a consistent technique. After induction of general anaesthesia, the patient was placed in a beach chair position and thorough examination of the affected shoulder was performed to assess the magnitude and direction of instability. The shoulder was then prepared and draped in a sterile manner, and the bony landmarks marked so that the orientation of the shoulder was maintained throughout the procedure. A standard posterior viewing portal was established approximately $2 \mathrm{~cm}$ inferior and $1 \mathrm{~cm}$ medial to the acromial angle. Two anterior portals were created with a spinal needle, using an outside-in technique to obtain an optimal distance between the portals. A small 5-mm cannula was inserted into the anterosuperior portal that was made in the rotator interval, while a larger 8-mm threaded cannula was inserted into the second portal

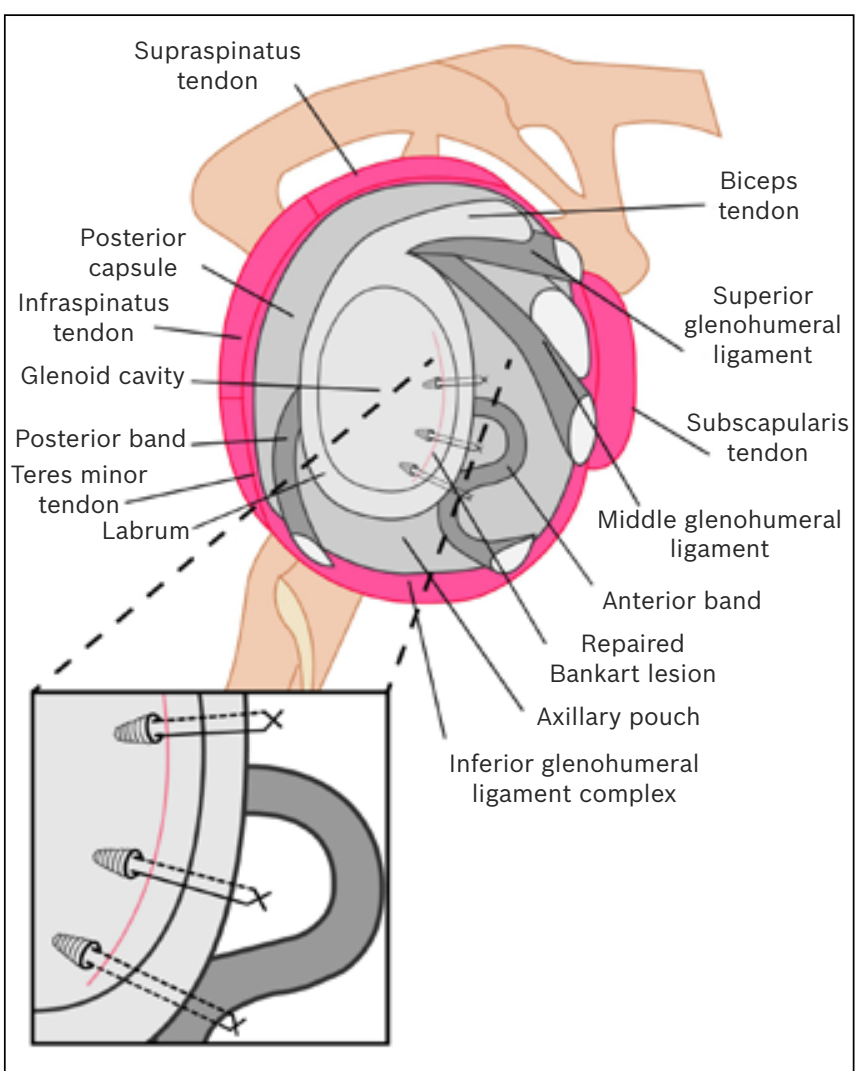

Fig. 2 Illustration shows the positions of the suture anchors in arthroscopic Bankart repair augmented by plication of the inferior glenohumeral ligament via horizontal mattress suturing.

which was created just above the superior border of the subscapularis. Together with a $30^{\circ}$ arthroscope placed in the posterior portal, the detached anteroinferior labrum was elevated with a periosteal elevator (which was inserted via the anteroinferior portal) and the surrounding degenerated tissues were debrided with a shaver.

Bioabsorbable single-loaded Lupine ${ }^{\mathrm{TM}}$ BR anchors (DePuy Mitek Inc, NJ, USA) were used. These anchors were placed sequentially, inferior to superior, at the 5:30, 4 and 3 o'clock positions for the right shoulder, and correspondingly at the 6:30, 8 and 9 o'clock positions for the left shoulder. The first anchor was placed at the 5:30/6:30 o'clock position on the glenoid surface, $3 \mathrm{~mm}$ away from the labrum. Although the most inferior suture should be placed at the 6 o'clock position, this is often not possible due to limitations in the placement angle. Drill holes were made on the articular surface along the detached labrum. A drill guide is helpful in enabling precise placement and spacing of drill holes. The lower most anchor was secured using horizontal mattress suturing to create a huge bumper with the anterior band of the IGHL, which was pulled up and tied together with the lower most anchor. Anchoring the lower most anchor is important as it reduces the shoulder joint volume and pushes the labrum up toward the glenoid socket, restoring labral height and augmenting the labral bump. The second and third anchors were placed at the $4 / 8$ and 3/9 o'clock positions and secured with simple vertical sutures (Figs. $1 \& 2$ ). 
Table I. Patient demographics $(n=75)$.

\begin{tabular}{lc}
\hline Variable & No. of patients \\
\hline Average age* (yrs) & $24.09 \pm 7.89$ \\
Gender & \\
Male & 73 \\
Female & 2 \\
Occupation type & \\
Outdoor & 49 \\
Sedentary & 26 \\
Mean duration to surgery* (wks) & $20.97 \pm 19.46$ \\
Operative findings & \\
Bankart lesion & 76 \\
Lax capsule & 35 \\
Chondrolabral lesion & 8 \\
SLAP lesion & 5 \\
Hill-Sachs lesion & 4 \\
Frayed/torn biceps tendon & 3 \\
Bony Bankart lesion ${ }^{+}$ & 1 \\
\hline
\end{tabular}

${ }^{*}$ Data is presented as mean \pm standard deviation. ${ }^{\dagger}<25 \%$ bony fragment left in situ. SLAP: superior labrum anterior-posterior

Immediate postoperative rehabilitation included placement of the operated shoulder in a sling for a duration of six weeks, with pendular exercises for the first three weeks and active forward flexion to $90^{\circ}$ for the next three weeks. Isometric rotator cuff exercises were also performed during the first six weeks to prevent shoulder atrophy. Full shoulder mobilisation was expected after six weeks. The patients were scheduled for regular follow-up sessions after the operation (on the 3rd, 6th, 12th and 24th months). During these follow-up sessions, the patients were assessed both clinically and using the University of California Los Angeles (UCLA) shoulder rating scale. Clinical assessment consisted of evaluations of the clinical strength of the supraspinatus, and the range of motion and postoperative function of the operated shoulders. Assessment of the external rotation of the shoulder was performed with the patient's arm in adduction. Data analysis comparing the pre- and postoperative UCLA scores and the range of external rotation were done using repeated measures analysis with pairwise comparisons. A p-value of $<0.05$ was taken to be statistically significant.

\section{RESULTS}

The mean age of the patients at the time of presentation was 24.09 (range 15-48) years, and the mean interval from initial presentation to surgery was five months. A majority of the patients were male (98\%), and $65 \%$ of all patients were involved in physically demanding occupations (e.g. military personnel and professional athletes), which could have increased the patients' susceptibility in sustaining recurrent Bankart injuries. The mean duration of follow-up was 28.7 months. The operative findings, summarised in Table I, reveal lax capsule as the most common associated pathology. There were no intraoperative complications such as axillary nerve injury, bleeding or compartment syndrome.

At 24 months after surgery, 55 out of the 75 (73.33\%) patients had returned to their premorbid activity levels, while the remaining 20 patients either had a recurrence of Bankart

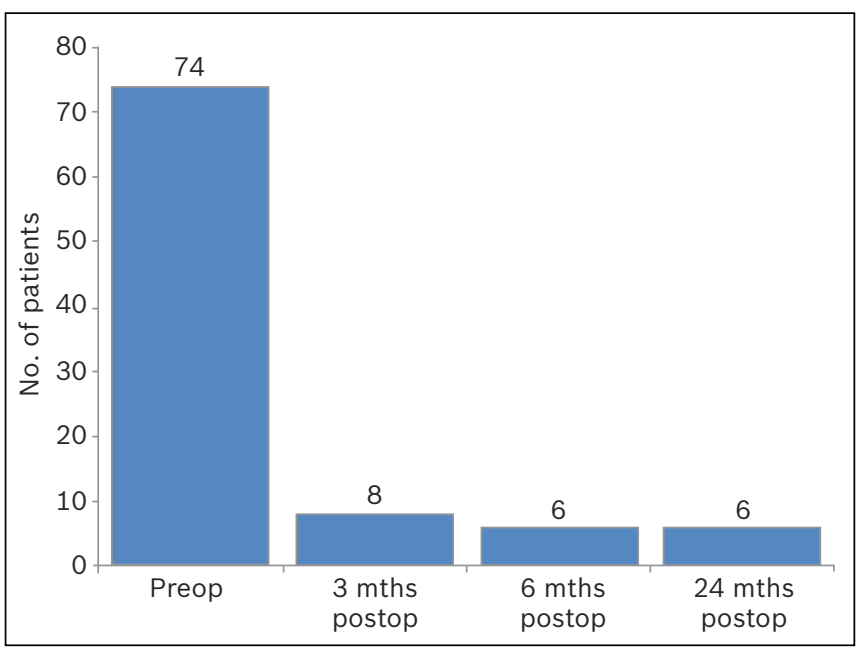

Fig. 3 Graph shows the number of patients who scored positively in the apprehension test before and after the augmented arthroscopic Bankart repair.

lesions or voluntarily restrained from sporting activities. The recurrence rate of dislocation was $7.89 \%$ (i.e. six shoulders), of which five shoulders eventually required revision surgeries (Fig. 3). All six patients had MR imaging performed on their affected shoulders. MR imaging showed that all six patients had recurrent labral tears with no bony glenoid or humeral involvement, and no suture pull-outs. Five of these six patients experienced recurrent symptoms after another episode of trauma while playing contact sports, while one dislocated his shoulder during sleep.

Significant improvements in the UCLA scores $(27.72 \%)$ were demonstrated six months after the operation, and these improvements were sustained for up to two years (Table II, Fig. 4). The stable repairs had no adverse effect on the range of motion of the operated shoulders (Table III, Fig. 5).

\section{DISCUSSION}

Currently, there is a myriad of methods of Bankart lesion repair for anterior glenohumeral instability. Various suturing techniques and augmented methods of arthroscopic Bankart repairs have been described in the current literature, including horizontal mattress suturing, closing rotator interval and capsular shrinkage. ${ }^{(9-12)}$

Hagstrom and Marzo conducted a cadaveric study analysing the difference between simple and horizontal suturing in restoring labral anatomy, and concluded that in vitro horizontal mattress suturing achieved better labral anatomy restoration. ${ }^{(12)}$ They also postulated that in clinical settings, horizontal mattress suturing may confer increased stability to glenohumeral repair. ${ }^{(12)}$ The importance of an intact labral and glenoid concavity in achieving shoulder stability was reinforced in another cadaveric study. ${ }^{(13)}$ In that study, which was conducted by Lazarus et al, a chondral-labral defect created in the cadavers resulted in the reduction of the height of the glenoid by approximately $80 \%$, and the reduction of the stability ratio by approximately $65 \%$ for translation in the direction of the defect. ${ }^{(13)}$ Interestingly, in 
Table II. UCLA outcome scores before and after the augmented arthroscopic Bankart repair $(n=76)$.

\begin{tabular}{lccr}
\hline Parameter & \multicolumn{3}{c}{ Mean \pm SD } \\
\cline { 2 - 4 } & Preop & 6 mths postop & 24 mths postop \\
\hline Pain & $6.27 \pm 1.87$ & $8.6 \pm 1.76$ & $8.9 \pm 1.65$ \\
Function & $7.9 \pm 2.55$ & $8.9 \pm 1.96$ & $9.5 \pm 1.79$ \\
Active forward flexion & $4.6 \pm 0.71$ & $4.8 \pm 0.55$ & $4.97 \pm 0.27$ \\
Strength of forward flexion & $4.3 \pm 0.61$ & $4.7 \pm 0.54$ & $4.8 \pm 0.35$ \\
Satisfaction of patient & 0 & $4.6 \pm 1.7$ & $4.9 \pm 1.33$ \\
Total & $25.94 \pm 3.43$ & $33.13 \pm 2.74$ & $33.77 \pm 3.07$ \\
\hline
\end{tabular}

Table III. Pairwise comparisons of the range of external rotation of the shoulder.

\begin{tabular}{lccccr}
\hline Time & $\begin{array}{c}\text { Range of external } \\
\text { rotation* }\end{array}$ & \multicolumn{3}{c}{ p-value } \\
\cline { 3 - 6 } & $78.10 \pm 8.77$ & Preop & 3 mths postop & $\mathbf{6}$ mths postop & 24 mths postop \\
\hline Preop & $63.97 \pm 20.52$ & - & - & 0.16 & 1.00 \\
3 mths postop & $74.84 \pm 11.11$ & - & - & -0.05 & $<0.05$ \\
6 mths postop & $79.52 \pm 3.78$ & - & - & - & $<0.05$ \\
24 mths postop & & & & - \\
\hline
\end{tabular}

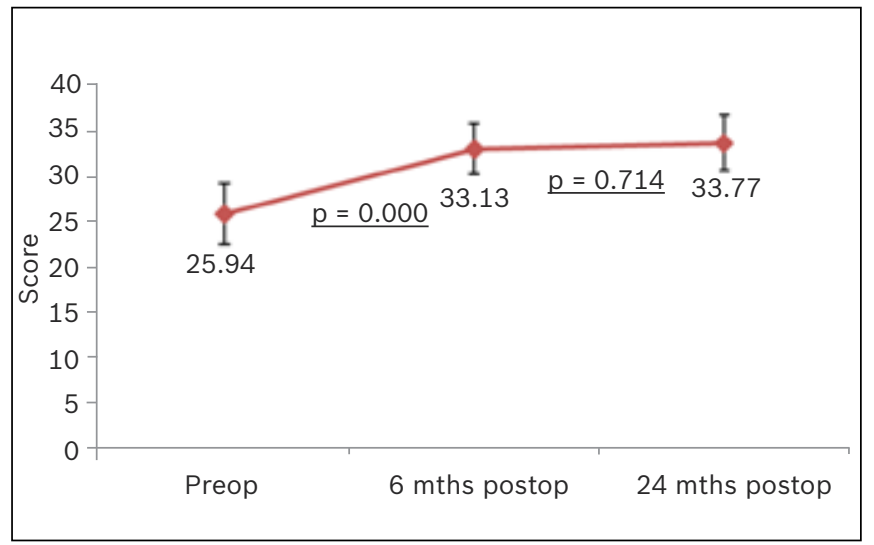

Fig. 4 Graph shows the University of California Los Angeles shoulder rating score of the patients before and after the augmented arthroscopic Bankart repair.

a biomechanical study by Nho et al that studied four different suture repair techniques (i.e. simple stitch, horizontal mattress stitch, double-loaded simple stitch and knotless anchor), the authors concluded that the four repair techniques performed similarly in vitro. ${ }^{(14)}$ Biomechanical studies analysing labral repair reinforced with the IGHL also showed that the labral repairs were secure without compromising the range of motion of the shoulders. ${ }^{(15,16)}$ The findings of these biomechanical studies provided an important platform for the current study, which aimed to optimise the current suturing repair technique.

Previously, the results of arthroscopic Bankart repair were shown to be inferior to that of open surgery. However, many of the early arthroscopic techniques described used transglenoid sutures or bioabsorbable tacks. ${ }^{(17,18)}$ Fortunately, recent new techniques that use suture anchor fixation and capsular plication have been shown to be effective and durable with promising results. ${ }^{(19)}$ As arthroscopic Bankart repair is minimally invasive, it has the potential to confer more advantages to a patient than repair using open techniques - namely reduced

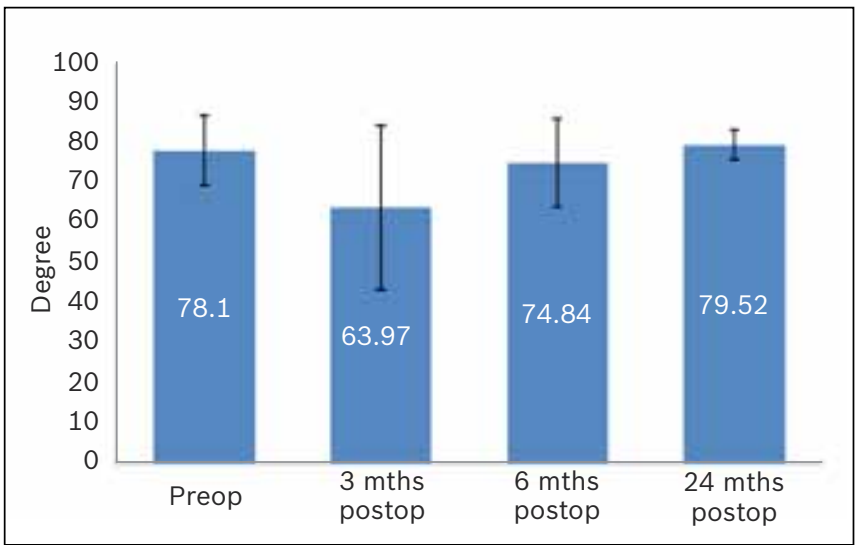

Fig. 5 Graph shows the mean range of external rotation of the shoulder before and after the augmented arthroscopic Bankart repair.

surgical trauma, blood loss, operative duration, perioperative morbidity, use of analgesia, length of hospital stay, time loss from work, number of complications and cost of surgery. ${ }^{(20)}$

In our study, the early improvement in functional outcomes reaffirm the benefits of arthroscopic shoulder repair, while the favourable long-term results highlight the viability and durability of the augmented arthroscopic Bankart repair, which could provide shoulder surgeons an additional safe option in managing Bankart lesions. However, there was concern that with this augmented arthroscopic Bankart repair technique, the shoulder's range of motion would potentially be sacrificed to achieve greater stability of the shoulder. Although this novel and augmented repair technique involved plication of the IGHL secured with horizontal mattress sutures, the mean external rotation of the operated shoulders was not observed to be adversely affected at the follow-up sessions 6 and 24 months after the operation. At the final follow-up on the 24th month, the mean range of external rotation was almost identical to that observed before the operation ( $p$-value in the 
pairwise comparison of external rotation before the operation and 24 months after the operation was 1.00). A satisfactory range of motion, especially external rotation, is essential for activities of daily living and rigorous sporting activities. A study by Fabbriciani et al reported a good range of motion after arthroscopic repair; in fact, the range of motion achieved after arthroscopic repair was sometimes better than that achieved with open Bankart repair. ${ }^{(17)}$

The recurrence rate in our study was $7.89 \%$, similar to other published clinical studies of arthroscopic Bankart repairs using simple stitch techniques; Gartsman et al reported a recurrence rate of $7.5 \%$, while Mishra and Fanton reported a recurrence rate of $7 \%$ with arthroscopic Bankart repair augmented by thermal capsulorrhaphy. ${ }^{(21,22)}$ These recurrence rates are consistent with the results from biomechanical studies that compared various suture techniques. ${ }^{(14)}$ In our study, favourable outcomes were observed with regard to the patients' return to premorbid physical activities $-73 \%$ of patients reported confidence in resuming their usual sporting activities. This result is similar to that of studies reporting on arthroscopic Bankart repairs using simple stitching with suture anchors; Ee et $\mathrm{al}^{(23)}$ and Seedek et $\mathrm{al}^{(24)}$ reported $75 \%-85 \%$ of their patients returning to their previous sporting activities.

Our study was not without limitations. Firstly, this study was a retrospective analysis of the clinical outcomes of a novel technique and not a randomised controlled trial. To further validate the efficacy of this technique, future randomised controlled studies may be warranted. Secondly, the 75 patients in this study were assumed to be a sufficiently large sample size to elucidate meaningful interpretations. Thirdly, since the UCLA score was used without the incorporation of other shoulder scoring indices such as the Rowe, Oxford or Western Ontario shoulder index, a comprehensive interpretation of the results of the novel technique may be lacking. The UCLA shoulder rating scale is a general scoring system for shoulder function, and unlike the Rowe, Oxford and Western Ontario Shoulder indices, it is not specific to shoulder instability.

In this study, we demonstrated that arthroscopic Bankart repair augmented by plication of the IGHL via horizontal mattress suturing is a viable alternative technique that shows promise in improving the outcomes of arthroscopic Bankart repair. We conclude that this novel technique is a safe and reliable treatment option for shoulder instability, with comparable clinical outcomes and recurrence rates to other reported arthroscopic techniques.

\section{REFERENCES}

1. Krøner K, Lind T, Jensen J. The epidemiology of shoulder dislocations. Arch Orthop Trauma Surg 1989; 108:288-90.

2. Zacchilli MA, Owens BD. Epidemiology of shoulder dislocations presenting to emergency departments in United States. J Bone Joint Surg Am 2010; 92:542-9.
3. Brophy RH, Marx RG. The treatment of traumatic anterior instability of the shoulder: nonoperative and surgical treatment. Arthroscopy 2009; 25:298-304.

4. Rowe CR, Patel D, Southmayd WW. The Bankart procedure: a long-term end-result study. J Bone Joint Surg Am 1978; 60:1-16.

5. Kim SH, Ha KI, Cho YB, Ryu BD, Oh I. Arthroscopic anterior stabilization of the shoulder: two to six-year follow up. J Bone Joint Surg Am 2003; 85-A:1511-8.

6. Coughlin L, Rubinovich $M$, Johansson J, White B, Greenspoon J. Arthroscopic staple capsulorrhaphy for anterior shoulder instability. Am J Sports Med 1992; 20:253-6.

7. Pagnani MJ, Warren RF, Altchek DW, Wickiewicz TL, Anderson AF. Arthroscopic shoulder stabilization using transglenoid sutures: a four year minimum followup. Am J Sports Med 1996; 24:459-67.

8. Dora C, Gerber C. Shoulder function after arthroscopic anterior stabilization of the glenohumeral joint using an absorbable tac. J Shoulder Elbow Surg 2000; 9:294-8.

9. Bahk MS, Karzel RP, Snyder SJ. Arthroscopic posterior stabilization and anterior capsular plication for recurrent posterior glenohumeral instability. Arthroscopy 2010; 26:1172-80.

10. Chiang ER, Wang JP, Wang ST, et al. Arthroscopic posteroinferior capsular plication and rotator interval closure after Bankart repair in patients with traumatic anterior glenohumeral instability-A minimum follow-up of 5 years. Injury 2010; 41:1075-8.

11. Westerheide KJ, Dopirak RM, Snyder SJ. Arthroscopic anterior stabilization and posterior capsular plication for anterior glenohumeral instability: a report of 71 cases. Arthroscopy 2006; 22:539-47.

12. Hagstrom LS, Marzo JM. Simple versus horizontal suture anchor repair of Bankart lesions: which better restores labral anatomy? Arthroscopy 2013; 29:325-29

13. Lazarus MD, Sidles JA, Harryman DT 2nd, Matsen FA 3rd. Effect of a chondral-labral defect on glenoid concavity and glenohumeral stability. A cadaveric model. J Bone Joint Surg Am 1996; 78:94-102.

14. Nho SJ, Frank RM, Van Thiel GS, et al. A biomechanical analysis of anterior Bankart repair using suture anchors. Am J Sports Med 2010; 38:1405-12.

15. Peltier KE, McGarry MH, Tibone JE, Lee TQ. Effects of combined anterior and posterior plication of the glenohumeral ligament complex for the repair of anterior glenohumeral instability: a biomechanical study. J Shoulder Elbow Surg 2012; 21:902-9.

16. Cueff F, Ropars M, Chagneau F, et al. Interest of complementary inferior glenohumeral ligament fixation in capsulo-labral repair for shoulder instability: A biomechanical study. Orthop Traumatol Surg Res 2010; 96 (8 suppl):S94-8.

17. Fabbriciani C, Milano G, Demontis A, et al. Arthroscopic versus open treatment of Bankart lesion of the shoulder: a prospective randomized study. Arthroscopy 2004; 20:456-62.

18. Freedman KB, Smith AP, Romeo AA, Cole BJ, Bach BR Jr. Open Bankart repair versus arthroscopic repair with transglenoid sutures or bioabsorbable tacks for recurrent anterior instability of the shoulder: a meta-analysis. Am J Sports Med 2004; 32:1520-7.

19. Rudzki JR, Purcell DB, Wright RW. Options for glenoid labral suture anchor fixation. Oper Tech Sports Med 2004; 12:225-31.

20. Wang C, Ghalambor N, Zarins B, Warner JJ. Arthroscopic versus open Bankart repair: analysis of patient subjective outcome and cost. Arthroscopy 2005; 21:1219-22.

21. Gartsman GM, Roddey TS, Hammerman SM. Arthroscopic treatment of anterior-inferior glenohumeral instability. Two to five-year follow-up. J Bone Joint Surg Am 2000; 82:991-1003.

22. Mishra DK, Fanton GS. Two-year outcome of arthroscopic bankart repair and electrothermal-assisted capsulorrhaphy for recurrent traumatic anterior shoulder instability. Arthroscopy 2001; 17:844-9.

23. Ee GW, Mohamed S, Tan AH. Long term results of arthroscopic Bankart repair for traumatic anterior shoulder instability. J Orthop Surg Res 2011; $6: 28$.

24. Sedeek SM, Tey IK, Tan AH. Arthroscopic Bankart repair for traumatic anterior shoulder instability with the use of suture anchors. Singapore Med J 2008; 49:676-81. 